\title{
Temperature Dependence of Magnetoimpedance in FeNi/Cu/FeNi Film Structures with Different Geometries
}

\author{
S.O. Volchkov ${ }^{2}$, D.A. Bukreevi,a , V.N. Lepalovskij ${ }^{2}$, A.V.Semirov ${ }^{1}$ \\ and G.V. Kurlyandskaya ${ }^{2}$ \\ ${ }^{1}$ East-Siberian State Academy of Education, Irkutsk, 664011, Russian Federation, \\ ${ }^{2}$ Ural State University, Ekaterinburg, 620000, Russian Federation; \\ abukreev_84@mail.ru
}

Keywords: magnetoimpedance, NiFe films, magnetic field sensor, MI temperature dependence

\begin{abstract}
The magnetoimpedance (MI) in thin films and magnetic multilayers has been extensively studied in recent years. One of the important problems which is still under discussion is the temperature dependence of MI of the multilayered nanostructures for temperatures up to $200{ }^{\circ} \mathrm{C}$. In this work magnetic properties and temperature dependence of the magnetoimpedance of $\mathrm{Fe}_{19} \mathrm{Ni}_{81} / \mathrm{Cu} / \mathrm{Fe}_{19} \mathrm{Ni}_{81}$ multilayers with opened and closed magnetic pathes were studied and comparatively analyzed for the functional temperature interval of $+23{ }^{\circ} \mathrm{C}$ to $+160{ }^{\circ} \mathrm{C}$.
\end{abstract}

\section{Introduction}

The magnetoimpedance effect (MI) is a change of the complex impedance of a soft ferromagnetic conductor under application of an external magnetic field [1-4]. The sensitivity of MI to a magnetic field was observed to be of the order of $500 \% / O e$ in amorphous ribbons [5]. However, in thin films the highest observed sensitivity did not exceed 35\%/Oe [6]. The studies of the temperature dependence of the MI effect in flat multilayered structures are rather limited [7]. Therefore, the purposeful research of the planar MI structures is necessary for better understanding of the physical processes proceeding in the soft magnetic materials with a high MI sensitivity in the conditions close to the temperature range of technological applications.

In this work, the magnetic properties and longitudinal MI were studied for the $\mathrm{Fe}_{19} \mathrm{Ni}_{81} / \mathrm{Cu} / \mathrm{Fe}_{19} \mathrm{Ni}_{81}$ multilayered elements with different geometries for a temperature range and frequency interval corresponding to the working areas for majority of the MI-based detectors.

\section{Experimental}

The $\mathrm{Fe}_{19} \mathrm{Ni}_{81} / \mathrm{Cu} / \mathrm{Fe}_{19} \mathrm{Ni}_{81}$ multilayered structures were deposited onto glass substrates at room temperature by rf-sputtering in the Ar atmosphere. The rectangular shape of the MI sensitive elements was obtained by metallic masks. Application of a magnetic field of about 100 Oe created by a calibrated system of the permanent magnets resulted in the formation of the in-plane induced magnetic anisotropy with the easy magnetization axis (EMA) oriented perpendicular to the long sides of the elements. The total length of all layers was $10 \mathrm{~mm}$. The width of non-magnetic layer was $0.2 \mathrm{~mm}$ for the samples with a closed magnetic flux and $0.5 \mathrm{~mm}$ for the samples with an opened magnetic flux (Fig. 1). The width of the ferromagnetic layers of $0.5 \mathrm{~mm}$ was kept constant in all cases. The detailed geometrical parameters for the samples are shown in the Table 1.

The geometry of samples was checked by optical microscopy and contact profilometry. The hysteresis loops were measured by magnetooptical Kerr-effect. The visualization of magnetic domain structure was done by the method of Akulov-Bitter in a zero external magnetic field using a commercial ferrofluid. The longitudinal MI (total impedance (Z), and its real (R) and imaginary (X) components) [5] was measured as a function of the external magnetic field by an automated device based on an impedance analyzer Agilent 4294 for the frequency range of 1 to $100 \mathrm{MHz}$ for a driving current of $1.5 \mathrm{~mA}$. The MI studies were done for the temperature range of $+23{ }^{\circ} \mathrm{C}$ to $160{ }^{\circ} \mathrm{C}$. The MI ratios were calculated as follow: $\Delta \mathrm{Z} / \mathrm{Z}=100 \times\left(|\mathrm{Z}(\mathrm{H})|-\left|\mathrm{Z}\left(\mathrm{H}_{\max }\right)\right|\right) /\left|\mathrm{Z}\left(\mathrm{H}_{\max }\right)\right|$, where 
$\mathrm{H}_{\max }=150$ Oe. The division into an active and reactive component of the impedance was presented in the frame of the given technique.
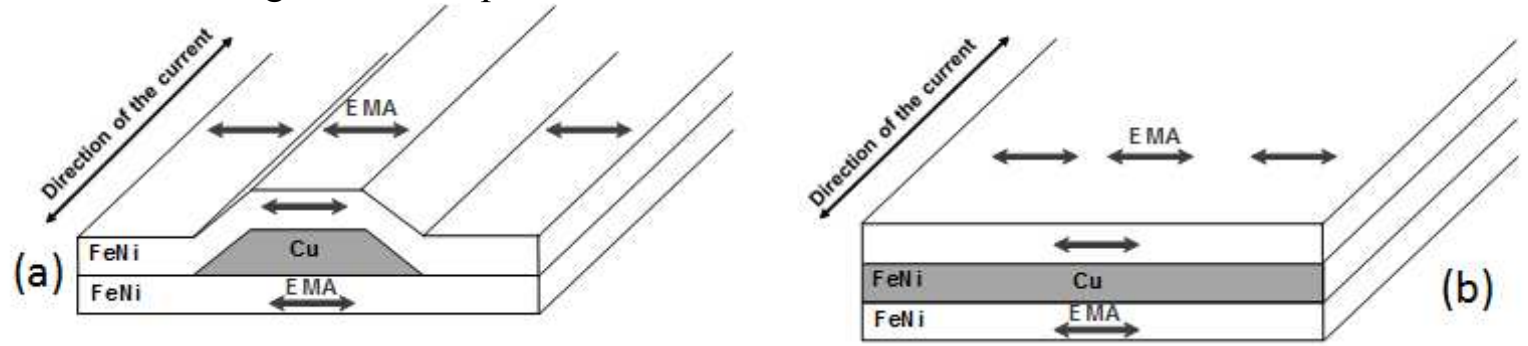

Fig. 1. The types of magnetoimpedance elements with open (a) and closed (b) magnetic flux.

Table 1. The geometrical dimensions of multilayered structures

\begin{tabular}{|l|l|l|l|l|}
\hline $\mathrm{N}$ & $\begin{array}{l}\text { Multilayered structure (The thickness of a } \\
\text { corresponding layer is specified) }\end{array}$ & $\begin{array}{l}\text { Width }(\mathrm{FeNi}), \\
\mathrm{mm}\end{array}$ & $\begin{array}{l}\text { Width }(\mathrm{Cu}), \\
\mathrm{mm}\end{array}$ & $\begin{array}{l}\text { Type of } \\
\text { magnetic flux }\end{array}$ \\
\hline $\mathrm{S} 1$ & $\mathrm{FeNi}(130 \mathrm{~nm}) / \mathrm{Cu}(500 \mathrm{~nm}) / \mathrm{FeNi}(130 \mathrm{~nm})$ & 0.5 & 0.2 & closed \\
\hline $\mathrm{S} 2$ & $\mathrm{FeNi}(130 \mathrm{~nm}) / \mathrm{Cu}(500 \mathrm{~nm}) / \mathrm{FeNi}(130 \mathrm{~nm})$ & 0.5 & 0.5 & open \\
\hline $\mathrm{S} 3$ & $\mathrm{FeNi}(75 \mathrm{~nm}) / \mathrm{Cu}(500 \mathrm{~nm}) / \mathrm{FeNi}(75 \mathrm{~nm})$ & 0.5 & 0.2 & closed \\
\hline $\mathrm{S} 4$ & $\mathrm{FeNi}(75 \mathrm{~nm}) / \mathrm{Cu}(500 \mathrm{~nm}) / \mathrm{FeNi}(75 \mathrm{~nm})$ & 0.5 & 0.5 & open \\
\hline
\end{tabular}

\section{Results and discussion}

The longitudinal hysteresis loops of S2, S3 and S4 are very similar to each other. The shape of the $\mathrm{M}(\mathrm{H})$ loops and magnetic domain structures of elements of all types confirms the fact of formation of the induced magnetic anisotropy with the EMA oriented in-plane and perpendicular to the long side of the rectangular element (Fig. 2(a). For the S2 and S4 samples a very low coercivity was observed and the value of the anisotropy field $\left(\mathrm{H}_{\mathrm{a}}\right)$ was estimated as approximately 5 Oe for $\mathrm{S} 2$ and 7 Oe for S4 samples, i.e. a rather reasonable value with respect to the possible technical applications. The shape of the hysteresis loop of the S1 and, to a certain extent ,S3 sample was rather complex (enhanced coercivity, high saturation field, existence of two types of magnetization process), leading to a conclusion about the existence of a «transcritical» state with the appearance of an out-of-plane magnetization component corresponding to the $\mathrm{FeNi} / \mathrm{FeNi}$ parts of the samples with the open magnetic flux $[5,6]$.
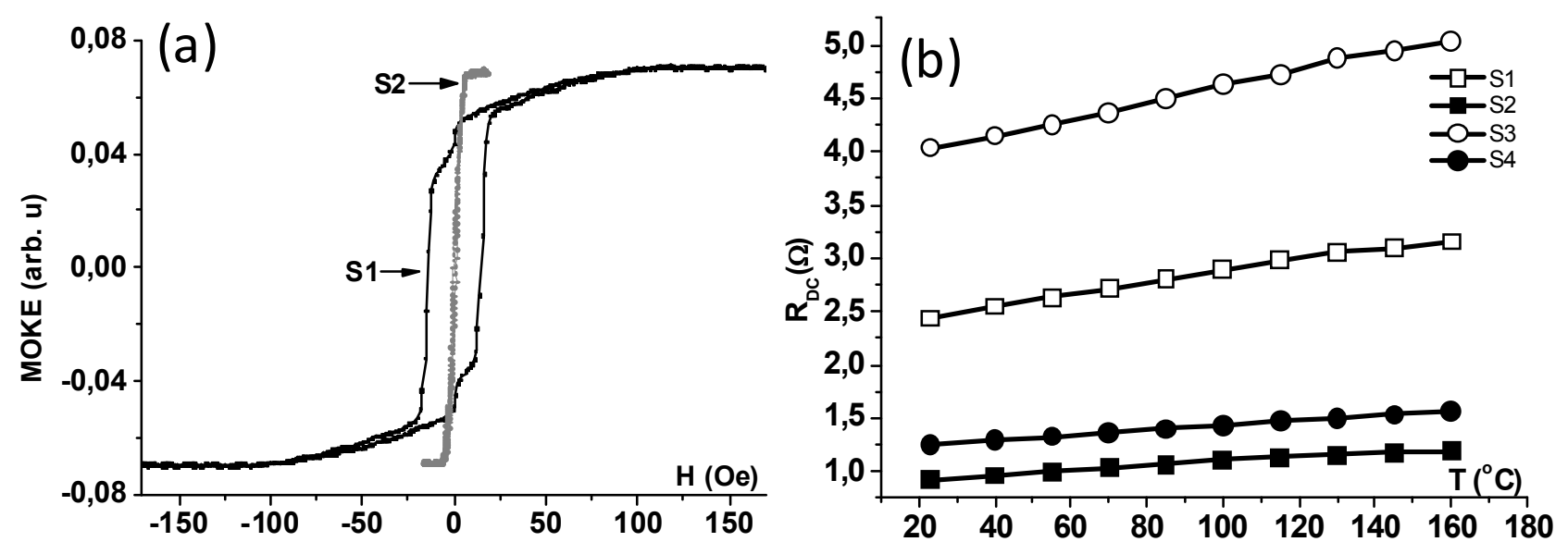

Fig. 2. Hysteresis loops for $\mathrm{S} 1$ and $\mathrm{S} 2$ elements (a). Temperature dependence of the DCelectroresistance of the film elements $\mathrm{FeNi} / \mathrm{Cu} / \mathrm{FeNi}$ at the direct current $1.5 \mathrm{~mA}(\mathrm{~b})$.

Fig. 2(b) shows the temperature dependence of the electrical resistance for a direct current (DC) of $1.5 \mathrm{~mA}$ for elements of all types. The resistance values showed the reversible linear increase with increasing the temperature. The difference of the value of the DC-resistance seems to be caused by variations of the geometry of the $\mathrm{Cu}$ conductive and FeNi ferromagnetic layers: the highest DCresistance value was observed for the S3 sample (with the smallest cross section); the smallest DC- 
resistance was observed for the S2 sample (with the largest cross-section). The behaviour of the impedance of the samples in the zero external magnetic field showed similar linear dependencies for small frequencies. The given values (up to $10 \Omega$ ) of the DC-resistance and AC-impedance of the MI samples are quite compatible with the semiconductor electronics.

Fig. 3(a) shows the frequency dependences of the MI-ratios of all samples. One can see that MI of the S1 and S3 samples with the closed magnetic flux shows a very strong dependence on the thickness of the magnetic layers: for the frequencies below $20 \mathrm{MHz}$ the MI ratios are rather similar and the difference between them becomes much higher with the frequency increase. In contrast, the frequency dependencies of the MI of the S2 and S4 samples with the open magnetic flux are very similar to each other over the whole frequency range under consideration. In the low-frequency range the samples with the open magnetic flux (S2, S4) show a slightly higher MI ratio comparing with the samples having the closed magnetic flux (S1, S3) in contrast to the usually described advantage of the closed flux structures (obtained by modeling [2]).
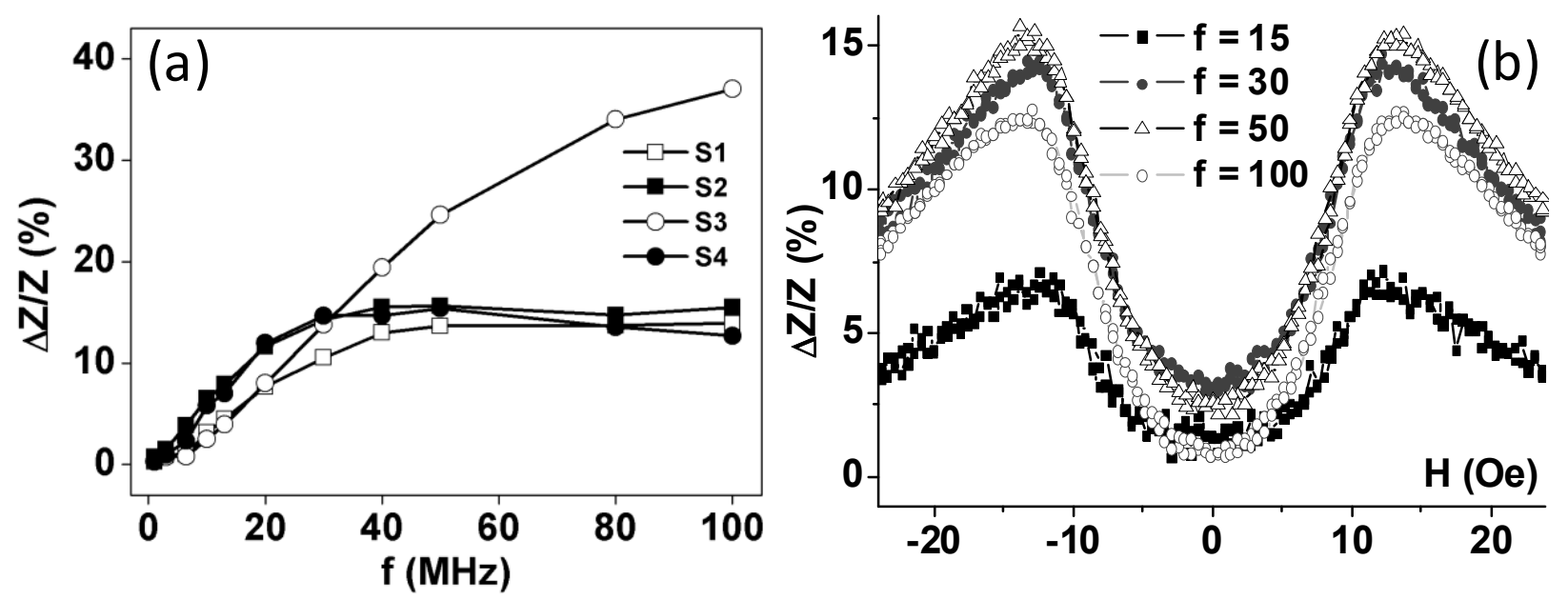

Fig. 3. (a) Frequency dependence of the maximum MI-ratio for the elements with different geometries for $\mathrm{T}=23^{\circ} \mathrm{C}$. (b) MI-ratio for the S4 sample for selected frequencies.

Fig. 3(b) shows the field dependences of MI for the S4 sample measured for selected frequencies. Although the maximum value of the MI ratio increases with a frequency increase, the maximum sensitivity, calculated as the MI ratio change per the unit of external magnetic field, $(\Delta \mathrm{Z} / \mathrm{Z})(\% / \mathrm{Oe})$ reaches a maximum at a frequency of $50 \mathrm{MHz}$. The $\mathrm{S} 4$ multilayered structure is the most simple with respect to the technology of structure preparation with smallest total thickness of the layers. Fig. 4(a) shows an example of the temperature dependence of MI for the S4 element with the open magnetic flux. It is important to mention the low-temperature dependence of the MI-effect (with a deviation below $0.5 \%$ ) in a low-frequency range of 0 to $40 \mathrm{MHz}$. The strongest temperature changes of the dependences $\Delta \mathrm{Z} / \mathrm{Z}=\mathrm{f}(\mathrm{T})$ are observed at the frequency $50 \mathrm{MHz}$ for the sample $\mathrm{S} 4$ (Fig. 4(b)). The obtain results can be useful to designers of the MI sensors.

\section{Summary}

The multilayered $\mathrm{FeNi} / \mathrm{Cu} / \mathrm{FeNi}$ sensitive elements with the open and closed magnetic flux and different thickness of magnetic layers were prepared by rf-sputtering. Their magnetic properties and MI were studied at different temperatures from $+23{ }^{\circ} \mathrm{C}$ to $+160{ }^{\circ} \mathrm{C}$. The $\mathrm{MI}$ of the $\mathrm{FeNi}(130$ $\mathrm{nm}) / \mathrm{Cu}(500 \mathrm{~nm}) / \mathrm{FeNi}(130 \mathrm{~nm})$ samples with the different thickness of magnetic layers show a very strong dependence on the thickness of the FeNi components: for the frequencies below 20 $\mathrm{MHz}$ the MI ratios are rather similar for the FeNi-sample thickness of 75 or $130 \mathrm{~nm}$, but the 

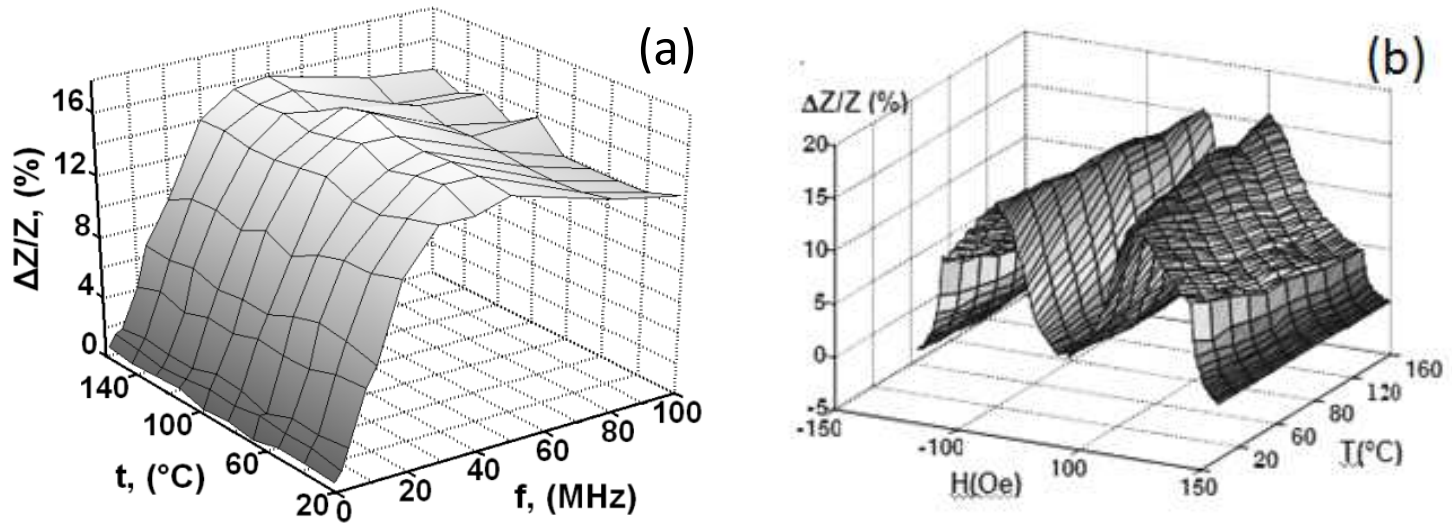

Fig. 4. Frequency dependence of the MI ratio on the temperature for the S4 element (a). The MI ratio for the frequency of $50 \mathrm{MHz}$ for the sample $\mathrm{S} 4$ (b).

difference between them becomes rather significant with the frequency increase. In contrast, the frequency dependencies of the MI FeNi(130 nm)/Cu(500 nm)/FeNi(130 nm) sample with the open magnetic flux are very similar to each other over the whole frequency range under consideration. In the low frequency range the elements with the open magnetic flux show the higher MI ratio, comparing with the elements with the closed flux. For small frequencies $(0-40 \mathrm{MHz})$ an admissible stability of the MI-effect upon temperature changing was observed.

\section{Acknowledgements}

We would like to thank Prof. V.O. Vas 'kovskiy and A.A. Moiseev for special assistance and helpful discussion. Supports by Grant RFBR №09-08-00406-a are acknowledged.

\section{References}

[1] R.S. Beach and A.E. Berkowitz, Appl. Phys. Lett., v.64, 3654 (1994).

[2] T. Uchiyama, K. Mohri, Panina L.V. and K. Furuno: IEEE Trans. Magn. Vol.31 (1995), p.3182.

[3] A. Antonov, S. Gadetsky, A. Granovsky, A. Diachkov, M. Sedova, N. Perov, T. Furmanova and A. Lagarkov: Physica A, Vol. 241 (1997) p. 414.

[4] S.O. Volchkov, A.V. Svalov and G.V. Kurlyandskaya, Solid State Phenomena Vol. 152-153 (2009), pp 373-376

[5] G.V. Kurlyandskaya, V. Fal Miyar, A. Saad, E. Asua, and J. Rodriguez, J. Appl. Phys., v. 101, 054505 (2007).

[6] D. de Cos, A. García-Arribas, and J. M. Barandiarán, Sens. Actuators A, vol. 115, pp. 368-375, 2004.

[7] Nishibe, Y., Yamadera, H., Ohta, et al., Sensors and Actuators, 2000, vol. 82, pp. 155-160.

[6] Svalov A.V., Kurlyandskaya G.V, Hammer H. et al., JTP, 2004, V.74.- №7.- P.62.

[7] S. O. Volchkov, V. N. Lepalovskii, A. V. Svalov, V. O. Vas'kovskii, and G. V. Kurlyandskaya Russian Physics Journal, Vol. 52, No. 10, 20091092-1097 